\title{
Quantum information \\ as the information of infinite collections or series
}

\author{
Vasil Penchev, vasildinev@.gmail.com \\ Bulgarian Academy of Sciences: Institute of Philosophy and Sociology: \\ Dept. of Philosophy of Science
}

\begin{abstract}
The quantum information introduced by quantum mechanics is equivalent to a certain generalization of classical information: from finite to infinite series or collections. The quantity of information is the quantity of choices measured in the units of elementary choice. The "qubit", can be interpreted as that generalization of "bit", which is a choice among a continuum of alternatives. The axiom of choice is necessary for quantum information. The coherent state is transformed into a well-ordered series of results in time after measurement. The quantity of quantum information is the transfinite ordinal number corresponding to the infinity series in question. The transfinite ordinal numbers can be defined as ambiguously corresponding "transfinite natural numbers" generalizing the natural numbers of Peano arithmetic to "Hilbert arithmetic" allowing for the unification of the foundations of mathematics and quantum mechanics.
\end{abstract}

Keywords: bit, entanglement, information, quantum information, qubit,

\section{INTRODUCTION}

The thesis is:

The quantum information introduced by quantum mechanics is equivalent to that generalization of the classical information from finite to infinite series or collections.

A few preliminary notes about the history of the problem are necessary:

The conception of quantum information was introduced in the theory of quantum information studying the phenomena of entanglement in quantum mechanics. The entanglement was theoretically forecast in the famous papers of Einstein, Podolsky, and Rosen (1935) and independently by Shrödinger (1935) deducing it from the separable complex Hilbert space, the basic mathematical formalism of quantum mechanics. However, the former three authors demonstrated the forecast phenomenon as the proof of the alleged "incompleteness of quantum mechanics". John Bell (1964) deduced a sufficient condition as an experimentally verifiable criterion in order to distinguish classical from quantum correlation (entanglement). Aspect, Grangier, and Roger (1981, 1982) confirmed experimentally the existence of quantum correlations exceeding the upper limit of the possible classical correlations. The theory of quantum information has thrived since the end of the last century in the areas of quantum computer, quantum communication, and quantum cryptography though directed rather to applications than fundamentally.

The unit of quantum information is the 'quantum bit', "qubit" definable as the normed superposition of any two orthogonal subspaces of complex Hilbert space as follows:

'Qubit' is: $\alpha|0\rangle+\beta|1\rangle$ where $\alpha, \beta$ are complex numbers such that, $|\alpha|^{2}+|\beta|^{2}=1$, and $|0\rangle,|1\rangle$ are any two orthonormal vectors (e.g. the orthonormal bases of any two subspaces) in any complex vector space (e.g. Hilbert space, Euclidean space, etc.). Thus, the separable complex Hilbert space space underlying quantum mechanics is representable as the quantity of quantum information and any wave function, i.e. any state of any quantum system being a vector ("point') in it can be seen as a value of that quantity. Consequently, all physical processes turn out to be quantum-informational in the final analysis, and nature or the universe can be interpreted as a single quantum computer processing 
quantum information.

The qubit is also isomorphic to a ball in Euclidean space, in which two points are chosen: the one chosen within the ball, and the other one being the orthogonal projection on its surface, i.e. as a mapping of a unit ball onto its surface (or any other unit sphere).

The main statement: Quantum information is equivalent to the generalization of information from finite to infinite series.

A sketch of the proof:

Indeed information can be interpreted as the number of choices necessary to reach an ordering of some item from another ordering of the same item or from the absence of ordering particularly. Then, the quantity of information is the quantity of choices measured in the units of elementary choice. A bit is that unit of elementary choice: it represents the choice between two equiprobable alternatives.

Furthermore, the unit of quantum information, the qubit, can be interpreted as that generalization of bit, which is a choice among a continuum of alternatives. Thus, it is able to measure the quantity of information as to infinite sets because any well-ordering of a certain infinite set can be interpreted as a state of it (and isomorphic to a quantum state if a well-ordering of an infinite set amd a qubit are proved to share a certain wave function).

The axiom of choice is necessary for quantum information in two ways: (1) in order to guarantee the choice even if any constructive approach to be chosen an element of the continuum does not exist; (2) to equate the definition in terms of Hilbert space and that as a choice among a continuum of alternatives:

Indeed, the theorems about the absence of hidden variables in quantum mechanics (Neumann 1932; Kochen, Specker 1968) demonstrate that the mathematical formalism of quantum mechanics implies that no well-ordering of any coherent state might exist before measurement. However, the same coherent state is transformed into a well-ordered series of results in time after measurement. In order to equate the state before and after measurement, the well-ordering "theorem" equivalent to the axiom of choice is necessary. Particularly, the measurement mediating between them should be interpreted as an absolutely random choice of an element of the coherent state, for which no constructive way (since it would be equivalent to some "hidden variable" otherwise) can exist in principle.

Thus, the quantity of quantum information can describe uniformly the state before and after measurement (equivalent to a choice among all elements of an infinite set). Thus, the separable complex Hilbert space can be understood as the free variable of quantum information. Then any wave function, being a given value of it, "bounds" an unorderable and a well-ordered state as the quantity of qubits (i.e. the "infinite choices") necessary for the latter to be obtained from the former.

Properly, the last statement underlies the establishment of the contemporary quantum mechanics as the property of unitarity allowing for Heisenberg's matrix mechanics and Schrödinger's ondulatory mechanics to be unified since the former interprets all elements of the separable complex Hilbert space as vectors (what the matrices are in the final analysis) equivalent to square-integrable functions (to the class of which all wave functions belong).

Further, Heisenberg's matrices can be understood as quantum states after measurement, and Schrödinger's wave functions as the same states, but before measurement. Then, the property of unitarity (for which, properly, the separable complex Hilbert space is the chosen basic mathematical structure of the "classical" quantum mechanics) allows quantum mechanics to restore both scientific objectivity and energy conservation even the same as in classical physics rather then essentially 
generalized to be valid only as particular cases of more fundamental laws.

1. As to scientific objectivity, the unification "resigns" the coincidence of the state before and after measurement: a principle of classical physics joining theories and experiments and often notated as the "principle of transparent measurement" postulating its property not to disturb any results of correct experiments, The Planck constant prevents quantum measurement to be also transparent as to a single measurement turning out to be fundamentally random: however, scientific objectivity can be restored to any infinite set of single measurements of the same quantum phenomenon: thus, that single, though infinite set of measurements can be interpreted as a single measurement, but on the metalevel and resulting in a certain wave function to which the classical scientific objectivity can be reinstated literally.

The Standard model steps further conceptually by the identification of "local and global space" (both being the same separable complex Hilbert space), now interpretable as the identification of a certain wave function (on the theoretical metalevel) and a corresponding physical entity (on the empirical or experimental level). Thus, the classical scientific objectivity is put back ultimately also literally: the same ontological essence can be observed in two complementary empirical "hypostases" by classical physics and quantum mechanics, as properly empirical in the former, but as both empirical and mathematical in the latter (therefore implying a new, quantum form of Pythagoreanism (Penchev 2021 August 24).

Then, quantum information allows for those two empirical "hypostases" to be identified to each other as well as to the ontological essence underlying both (Penchev 2021 July 26). The understanding of quantum information as the information of infinite sets establishes the paradigm of the Standard model for identifying the local and global space to be valid in theory of information as well.

2. As to energy conservation, the unitarity of the separable complex Hilbert space (allowed the unification of matrix and ondulatory mechanics, in particular) implies it (Penchev 2020 October 5). However, this deduction can be interpreted as a corollary from a more general law: quantum information conservation. Then, it is able to regules all the phenomena of entanglement (conjecturally, observed experimentally as "dark matter" and "dark energy": Penchev 2021 October 20 ), to which energy conservation as well as the universality of space-time are not valid in general, rather than the particular case of phenomena observed on the screen of space-time, obeying energy conservation (or energy-momentum conservation in general relativity) and postulated to be all claiming to be physical not only by classical physics and relativity, but even by the "classical" quantum mechanics dominating recently and neglecting implicitly that entanglement needs and implies a relevant reinterpretation of the foundations of quantum mechanics.

The understanding of quantum information as the information of infinite sets and series allows for entanglement to be the situated in the conceptual framework of the Standard model (and thus, of the "classical" quantum mechanics) as describing the gradual transition from the local space to the global space (both being the same separable complex Hilbert space, infinitely dimensional in general) therefore referable to a transfinite domain between the finite and countably infinite one (its elements are neither finite nor countably infinite, being moreover greater than the former, but less than the latter).

The quantity of quantum information and its values are those transfinite ordinal numbers (Penchev $2005)$ corresponding to the sets and infinite series in question. Both definitions of "ordinal number" 
(Cantor 1897; Neumann 1923) are applicable as the ordinals are "small" being less than any countable ordinal number. The definition of ordinal number in Cantor - Russell refers to a class consisting of the factorial of the ordinal numbers defined in Neumann one-to-one to a coherent state (and here, as the quantity of quantum information contained in it).

The paper is organized in five sections. Section 2 introduces the necessary notions and general viewpoint, in which the main statement is obvious. Section 3 offers the proof and comments on the main statement in detail. Section 4 clears up its interpretation in a few relevant reference frames: set theory, foundation of mathematics, theory of information and quantum mechanics. Section 5 presents the conclusions and provides directions for future work.

\section{THE NOTIONS AND GENERAL VIEWPOINT}

One can consider the following concepts as relevant to the focus of the paper: information; quantum information; information as a binary series (following Kolmogorov's conception of information very often named "complexity"); choice; (quantum) information as the quantity of choices; the axiom of choice; a choice from infinity (more precisely from the elements of an infinite set); the separable complex Hilbert space (equivalent to the qubit Hilbert space under a few additional conditions); coherent state mappable equivalently into a relevant statistical ensemble; and quantum mechanics as a theory of quantum information. These concepts will be discussed in more detail below:

"Information" is a very wide and thus undetermined concept. However, its fruitfulness relies just on this to some degree.

It means some relation between orders or orderings. These relations and orderings can be both qualitative and quantitative. Only the latter case is meant in this paper.

The usual quantitative definitions of information refer to the mathematical relation of entropies or as some modification or generalization of the quantity of entropy. Indeed, entropy can be interpreted as a measure of disorder, therefore as equivalent to the opposite (or anti-isometric) of the measure of order as what information is considered.

Thus the quantity of information turns out to refer to some statistical ensembles, respectively, to the change of a single one.

Shannon's (1948) definition of information only interprets the members of the ensemble as messages translatable in communication channels :

The significant aspect is that the actual message is one selected from a set of possible messages. The system must be designed to operate for each possible selection, not just the one which will actually be chosen, e.g. since this is unknown at the time of design (Shannon 1948: 379).

The especially valuable idea demonstrates that a common and even mathematical concept underlies both areas addressing techniques and nature. A bridge is outlined between the natural and artificial realms. Similar viewpoints seem to be "floating in the air" in MIT then after Shannon's colleague Norbert Wiener (1948) had published the book "Cybernetics: Or Control and Communication in the Animal and the Machine", become famous subsequently.

\footnotetext{
${ }^{1}$ The definition of ordinal number in Cantor - Russell refers to a class consisting of the factorial of all well-orderings sharing the same ordinal. Though that class is too large (and thus, is cardinal number can be inaccessible in the framework of set theory, in general), it is taken as a whole looking as if that factorial is reduced as the common factor in both numerator and denominator of the fraction (as to Neumann's "ordinal number" in comparison to Cantor - Russell's definition) The latter definition refers to classes consisting of the "factorial of infinity" of elements (too wide in general in comparison with those admissible according to ZFC set theory): but this can be round and thus avoided in Neumann's way.
} 
Other papers (Penchev 2021 February 25; Penchev 2020 December 14) extend the same "cybernetic synthesis" to the foundation of both nature (quantum mechanics) and mathematics (set theory) complementing a neo-Pythagorean nuance (Penchev 2021 August 24) to that impressing general science of cybernetics or theory of information.

One can add that entropy and therefore information can be interpreted as a particular case of expectation or mathematical expectation, just that of the expectation of a probability distribution.

"Quantum information": Though information as above can be defined for any real functions, generalized to complex ones, and even so on, all this remains mainly a mathematical exercise for all those generalizations that are not necessary for the solutions of experimental or empirical, scientific or technical problems.

However, the generalization of information, to which quantum mechanics is forced in order to be able to be reformulated in terms of information, is necessary for the solution of its basic problem: how to be unified and then uniformly described quantum leaps, i.e. discrete morphisms, and classical motions, i.e. smooth morphism differentiating from each other by the availability of velocity in any point of the trajectory only in the latter case.

The solution turns out to involve necessarily infinity directly into an experimental science such as quantum mechanics as far as the above unification is impossible for any finite mathematical structure. So, the separable complex Hilbert space, which just underlies quantum mechanics, is not only infinitely dimensional in general, but furthermore just being complex, it requires a continuum to be defined even for any finite set of dimensions.

Thus quantum information introduced by quantum mechanics is not only a mathematical puzzle (in fact, not too difficult), but necessarily referring to infinity to resolve its problem:

Quantum mechanics turns out to be the first and probably single experimental science utilizing infinity: it is able to make experiments referring to infinity postulated to be empirically accessible by it in the final analysis.

Accordingly, quantum information can be equivalently interpreted as the quantity of choices, each of which is among an infinite set of alternatives in general. The generalization from classical to quantum information by their corresponding units of elementary choice, from a bit (as a "finite choice") to a qubit (as an "infinite choice"), is necessary for the solution of the main problem of quantum mechanics.

"Information as a binary series": Kolmogorov (1965) offered a fundamentally different understanding of information and then a new quantitative definition turned out to be equivalent to the entropic one in some degree ${ }^{2}$ (Kolmogorov 1968).

If information is some relation of orders or orderings, it can be realized as the reordering from one to other(s). The reordering is an algorithm as an unambiguous way to construct the latter(s) starting from the former, which is the "initial conditions" of the problem.

The starting point can be also the "absolute chaos and disorder" or the "homogeneous chaos"

\footnotetext{
${ }^{2}$ The algorithmically defined information (equivalent to Kolmogorov's complexity) turns out not to be a reflexive relation unlike its entropic counterpart. However this is rather obvious because any choice is entropically irreversible unlike energy and thus similar to temperature. Indeed the entropy of the elementary choice of a bit is 1 , and the entropy in the opposite direction, i.e. the value of a bit to be erased, is 0 . Furthermore, a binary string A to be "revamped" into another B means the string A to be undone $x$ cells (without any entropic expense) and then redone $y$ cells (with some nonzero entropic expense). Consequently, the algorithmic information is non-reflexive in general for the $|x-y| \neq 0$.
} 
(Wiener 1938) shared by all possible orderings as a common "reference frame", any ordering should correspond one-to-one to some algorithm, which is able to create just this order from that "absolute chaos".

Further, any algorithm is equivalent to a certain Turing machine, which in turn can be unambiguously represented as a binary series, supposedly finite for the corresponding algorithm and Turing machine are to be finite for "calculation" according to common sense.

However, if quantum information refers to infinity in the sense above, the Kolmogorov definition seems to be to be generalized both to transfinite sequences and to series of transfinite ordinal numbers. However, there is not an obvious way for that meaningful generalization at first glance. The main statement of this paper will attempt to resolve the problem about that generalization.

It is worth adding that the Kolmogorov definition interprets the concept of order as well-ordering in the rigorous mathematical sense of the latter term, therefore involving directly the axiom of choice as to infinite series or sets in virtue of its equivalence to the well-ordering "theorem". Thus, the Kolmogorov algorithmic definition of information as "complexity" of sets or series whether finite or infinite explores the fundamental equivalence of "order" and "choice" (admissibly valid for them even as philosophical categories) rewriting it into terms of the former rather than of the latter as it is established initially (e.g. by Shannon's definition of information).

That more general understanding of information relying on both "order" and "choice" refers directly to quantum mechanics (and thus, to quantum information) since it underlies its establishment after the unification of matrix and ondulatory mechanics in virtue of the unitarity of the separable complex Hilbert space (discussed above). It relates to the proven completeness of quantum mechanics (for the absence of hidden "variables" in it), and can be utilized to the foundations of mathematics (Penchev 2020 August 25) and even to those of philosophy (Penchev 2021 August 24; Penchev 2021 July 26).

"Choice": Though both information and quantum information can be understood and defined as a relevant mathematical relation of orders, they can be equivalently represented as the quantities of elementary choices, correspondingly bits and qubits.

"Choice" and "order" turn out to be equally rigorous and precisely determinable concepts and thus equally relevant as the ground of "information". Both entropic and Kolmogorov definitions of information transform the concept of (dis)order in that of choice or vice versa:

The entropic definition uses the meditation of "probability" for the "probability" can be interpreted as the ratio of the choices of favorable alternatives to the choices of all alternatives instead of the ratio of the favorable ones to all.

The Kolmogorov definition is equivalent to a series of Turing sells, in which can be recorded either " 1 " or " $0 "$ " Thus, the collection of an empty cell and both alternatives recordable in it is an elementary choice, a bit of information.

"Choice" and "well-ordering" are closely related and even equivalent to each other in a mathematical sense. Thus the transition from "order" to "choice" means a transition to the duality of "(dis)order" and "well-ordering" in the final analysis.

"Choice as the "atom" of the course of time":

Though "choice" can be specified in an abstract way absolutely independent of "time", it can also be interpreted as an "atom" of time unifying the minimally possible "amount" of the three ways ("tenses") of time: the future, the present, and the past. For example, the future can be symbolized by the "empty cell" of the choice, the present, by the action of choice itself, and the past, by the chosen alternatives. 
One can add that the choice as to the physical course of time is "infinite" for quantum information measurable by units of "infinite choice" (qubits) is what underlies nature.

"Both quantum information and information are the same quantity of choice": Indeed, the only difference between classical and quantum information consists in the units of measurement, correspondingly bits or qubits.

"The axiom of choice": Once the choice is related to infinity, one needs the axiom of choice for it to be guaranteed even in the cases where any constructive way to be made the choice is not known or cannot exist in principle. Some constructive way to make a choice among the elements of any finite set exists always. The axiom of choice distinguishes (repetitive ${ }^{3}$ ) constructiveness from choice as to infinity. Thus, its sense might be expressed so: the concept of choice can be generalized from finiteness to infinity at the expense of the loss of (repetitive) constructiveness, which loss in turn can be interpreted as "randomness". Moreover, one can demonstrate that quantum mechanics needs the axiom of choice:

Indeed the theorems about the absence of hidden variables in quantum mechanics (Neumann 1932; Kochen, Specker 1968) demonstrate that the mathematical formalism of quantum mechanics implies that no well-ordering of any coherent state might exist before measurement.

However, the same coherent state is transformed into a well-ordered series of results in time after measurement. In order to equate the state before and after measurement, the well-ordering "theorem" equivalent to the axiom of choice is necessary. The quantum measurement mediating between them should be interpreted as an absolutely random choice of an element of the coherent state (i.e. a certain state among the superposition of possible ones), for which no constructive way (equivalent to some "hidden variable") can exist in principle ${ }^{4}$.

"A choice from infinity": The axiom of choice has a series of "ridiculous" corollaries just as quantum mechanics implies not less "ridiculous" ones, though. One of those "peculiarities" is the statement that any (incl. infinite) set of choices from infinity is equivalent to a single one just as any (incl. infinite) set of qubits is equivalent to a single one.

The separable complex "Hilbert space" (or the qubit Hilbert space) is the basic mathematical structure underlying quantum mechanics. It possesses a series of remarkable properties, a few of which are enumerated below:

1. It synthesizes arithmetic and geometry, positive integers and Euclidean space, which is the usual three-dimensional space of our experience.

2. Its elements are solutions to the main problem of quantum mechanics: how to be united and describe uniformly quantum leaps and smooth trajectories.

3. Its elements can be interpreted both as future states and as actual states of some quantum

\footnotetext{
${ }^{3}$ One can ascribe unique constructiveness (thus finite) to the choice of an element from an infinite set: in other words, an element can be determined after an algorithm consisting of a finite number of steps, but if that algorithm would be repeated, it would result differently in general, which property can satisfy a probabilistic algorithm: a probability distribution and a wave function as its characteristic function can be assigned immediately and consistently. This is still one reason for transfinite ordinal (natural) numbers to be identified with wave functions unambiguously in Hilbert arithmetic.

${ }^{4}$ The same idea can be utilized to prove that there exist problems which are not $\mathrm{P}$ calculable, but simultaneously they are NP calculable (in terms of the "P vs NP" problem). For example, the problem to guess the state of "Schrödinger cat" is not $\mathrm{P}$ calculable (because if it was $\mathrm{P}$ calculable, this would imply "hidden variables in quantum mechanics"), being simultaneously NP calculable trivially as a choice among two possible states (Penchev 2021 August 2021).
} 
system.

4. It coincides with the space dual to it. Thus the elements of the dual space are identical, but complementary (incommensurable simultaneously) to those of the initial Hilbert space.

5. Though the description by Hilbert space needs only the "half" of variables in comparison to classical mechanics, any "hidden variables" cannot be added in principle. It is inherently complete.

"Wave function" is a term coined by quantum mechanics to designate the elements ("points") of the separable complex Hilbert space as states of some quantum systems. The meaning of the term is easily extendable to any interpretations of that kind of Hilbert space and its elements such as the quantity featuring each infinite set unambiguously or the values of quantum information.

That extended meaning addresses certain ontological conclusions relating to nature, mathematical structures, and information (e.g. as in: Penchev 2021 July 26 or Penchev 2021 August 24).

"Coherent state as an equivalent of a statistical ensemble": The designated " 3 " property of the separable complex Hilbert space above implies that any coherent state interpreted as a fure one is equivalent to the corresponding collection of all present already measured states of the system. Thus, if time is only continuous (without any corresponding Hermitian operator and thus unlike any other physical quantity), this implies the equivalence of a coherent state (before measurement) to a relevant statistical ensemble (after measurement); then, the property of unitarity valid for the separable complex Hilbert space and the "conservation of energy conservation "in the "classical" quantum mechanics (Penchev 2020 October 5).

"Quantum mechanics as a theory of quantum information": Quantum mechanics can be exhaustedly rewritten in the language of quantum information by utilizing the qubit Hilbert space (equivalent to the separable complex Hilbert space under additional conditions). This means that the basis of nature is information and nothing else: there is nothing in the world besides quantum information! Consequently, the general viewpoint in the paper can be featured as grounded only by information in the final analysis:

Information is a universal and fundamental foundation in a series of scientific fields as the quantity of choices (Penchev 2021 February 25, etc). Choice underlies both physics as the "atom" of the course of time (Penchev 2020 December 14) and mathematics as the "atom" of any mapping . It is furthermore a "tool" for infinity to be described in detail quantitatively rather than qualitatively. In practice, this means that any infinite set can be distinguished from any other by its "wave function", which is a much more precise and rigorous description than the usual set-theory one by its ordinal or cardinal number (Penchev 2200 August 25). One can say that the quantity of quantum information is what can differentiate the infinite sets from each other for the "wave functions" are the different values of it according to the pathos of the present paper.

The main problem is how to be mathematically linked the following two cognitive entities to each other unambiguously: (1) the new description of any infinite set by a value of the quantity of quantum information, which is a wave function, and (2) the usual set-theory description by an ordinal number being or representable as a transfinite binary series in general. Hilbert arithmetic (furthermore, allowing for a philosophical and Pythagorean interpretation: Penchev 2021 August 24) is able to resolve this problem and thus, to unify the foundations of quantum mechanics and mathematics. 


\section{THE PROOF OF THE MAIN STATEMENT ACCOMPANIED BY A FEW COMMENTS}

Statement 1: Quantum information is equivalent to the generalization of information from finite to infinite sets or series.

The equivalence requires for quantum information to be interpretable boh as the information of infinite series and vice versa. Another way is to demonstrate the necessary existence of a one-to-one mapping of the separable complex Hilbert space and a relevant subset of transfinite series: in fact, that relevant subset is that to which the definitional validity of the axiom of choice refers. The validity is defined wider, the relevant subset is larger.

The usual interpretation of the axiom of choice tends to equate bijectively only any infinite set of any power (determined by a certain cardinal number) to a countable set. However, Thoralf Skolem (1922) demonstrates that the set-theoretical definition of finiteness (as linked and opposed to Dedekind's definition of "infinite set" by the existence of a bijection of the set at issue and its true subset) allows for the action of the axiom of choice to be extended to that kind of "Dedekind finiteness" rather than only to countable sets (and thus, the "relativity of the concept of set" using his words).

An infinite set can be mapped bijectively into a finite set (rather paradoxically at first glance) by virtue of the absolute (fundamentally non-constructive) randomness of the axiom of choice. Thus, two different "experiments" to choose all elements of a certain infinite set can consist of two finite sets of choices (respectively, a finite quantity of bits or classical information), but different finite sets in general and unlike the case to be chosen all elements of a finite set (the two sets of choices will consist of the same number of elements in the latter case).

Thus, the absolute randomness of the axiom of choice admits the bijective mapping of an infinite set into a finite set under the condition for the bijection at issue to be different in general after any application of the axiom of choice to that infinite set. A probability (eventually, density) distribution can describe exhaustively all possible applications of the axiom of choice to that infinite set: then, the characteristic function of the probability (density) distribution will be an element of the separable complex Hilbert space and thus, isomorphic to a wave function describing necessarily some quantum state of some quantum system.

Consequently, Statement 1 is trivial in the range of the axiom of choice once its interpretation is extended to the "Dedekind finiteness" (defined by the absence of any constant bijection of an infinite set to its true, being finite, subset). Any way for that mapping to be constructed or exemplified is not and cannot be demonstrated once the axiom of choice is what is utilized, though. Only those remarks and comments referring to the explicit formulation of that mapping are especially valuable even though not being any constructive proof about that existence.

The first remark allots a special property of Hilbert space: it is intrinsically "invariant to the axiom of choice", which can be demonstrated by means of its interpretation in terms of quantum mechanics.

Indeed, any element of it ("point") can describe equally well both states of a statistical ensemble, therefore well-ordered and corresponding coherent state "by itself" (i.e. before measurement) therefore unorderable always and in principle ${ }^{5}$. The validity of both descriptions implies (1) the well-ordering "theorem" equivalent to the axiom of choice while: (2) only the latter state (detached) excludes it.

\footnotetext{
${ }^{5}$ This is equivalent to the property of unitarity valid to the separable complex Hilnert space and allowing for the unification of matrix and ondulatory mechanics as the "classica;" quantum mechanics obeying energy conservation.
} 
Thus, the unity of (1) and (2) in turn implies the invariance to the axiom of choice as to any element of Hilbert space therefore interpretable as a transfinite well-ordering and as the quantity of the elements of the set "before that well-ordering" (and even excluding it in principle).

The second remark addresses the fact that the above "duality" as to the axiom of choice can be directly referred to the inherent duality of Hilbert space: It coincides with its dual space though being complementary in virtue of isometry or anti-isometry.

Consequently, if one means any element of it, this element is interpretable according to the just now involved duality above both as the length of a transfinite series corresponding to a statistical ensemble and as the coherent state corresponding to the statistical ensemble as before and after the well-ordering (fganted to be "measurement" in quantum mechanics).

The third remark notices that any "point" in Hilbert space (i.e. any "wave function") indicates unambiguously just one transfinite series or set therefore being able to serve both as its "name" and as the "quantity of infinity" unlike "infinite" as quality (not allowing to be distinguished two infinite sets or series even in set theory if both share the same cardinal number).

Consequently, the cherished constructiveness of the mapping of quantum information and the information of infinite series is achieved in a rather surprising way: any way for the transfinite set or series to be named unambiguously is necessarily constructive and exhaustedly describable by means of its name, i.e. by the point of the separable complex Hilbert space in the case in question. However, any indication about the unambiguous well-ordering of them is not possible just because of the duality to the axiom of choice and thus to the well-ordering.

One can utilize the metaphor of ordering some "much" into some a corresponding "many". Any ordering will be constructive for the results (any given "many" will be a result) are able to be one-to-one mapped into the set of all those orderings as they cannot depend on that "much" in principle just in virtue of its nature to be merely "much" (a quality unlike the different quantity of "many" comparable with each other).

Still one metaphor can offer a way for that special kind of constructiveness to be visualized: a "Rorschach spot". It can be seen so or otherwise, e.g. as "two horses" or as "a single butterfly". Each seeing as a given "something" is constructive as the seeing of the something is determined only by the seeing rather than by the Rorschach spot itself. Unlike this, the random naming even of the same something is not constructive being irreproducible in principle. Analogically, that choice guaranteed by the axiom of choice is random, irreproducible and unconstructive in principle. However, "seeing" or "naming" the same selected element being already reproducible is constructive.

That constructiveness depends on some choosing or convention crucially, though. This underlies the rather "ridiculous" dilemma between "random objectiveness" and "objectiveness depending on an observer" in quantum mechanics. That "constructiveness" can seem rather fraudulent if one does not reminisce that the same "fraud" underlies nature according to quantum mechanics ${ }^{6}$. The indication of things cannot help but be constructive even only in definition. Although our indications can be only a finite set, no reason for that constructiveness not to be generalized as to any infinite sets of indications.

\footnotetext{
${ }^{6}$ Rejected by Einstein by his famous aphorism that God does not play dice (first mentioned in his letter to Max Born: Einstein 1926)
} 


\section{INTERPRETATIONS}

There are at least ${ }^{7}$ four fields of interpretation of the main statement: set theory, the foundation of mathematics, information theory, and quantum mechanics. They will be considered one by one in detail:

Set theory can be interpreted as a mathematical doctrine of infinity. The concept of infinity is its core. However, the quantities assigned to infinity such as ordinal and cardinal numbers are rather unspecified and not able to feature distinguishably an infinite set from another. For this purpose, any method for ascribing an exactly determined function to any infinite set is especially valuable.

The main statement above means just this: the separable complex Hilbert space and all "points" in it can be assigned to infinity as a set of discerning "names" for all infinite sets. This is a method for the doctrine of infinity in mathematics to be transformed from a rather qualitative (though mathematical: axiomatic and deductive) doctrine into a rigorous quantitative theory directly applicable in a series of areas.

Indeed, the separable complex Hilbert space (or better, the qubit Hilbert space ${ }^{8}$ ) can be seen as an immediate generalization of Peano's arithmetic as follows:

The qubit Hilbert space is a free variable of quantum information as a well-ordered series of empty qubits. Any point in it (i.e. any "wave function") is a value of it therefore transforming it into a bound variable.

The $\mathrm{N}^{\text {th }}$ qubit can be interpreted as a "geometrical" generalization of the positive integer $\mathrm{N}$ after substituting the point "N" with the unit ball " $\mathrm{N}$ ". Thus, all values of this qubit "N" correspond to the single point, "number N" after the "arithmetical degeneration" collapsing the qubit into a point.

Consequently, arithmetic and geometry turn out to be inherently linked in the separable complex Hilbert space (due to the qubit Hilbert space attachable to it) for the former can be considered as an infinite-dimensional generalization of the usual Euclidean three-dimensional geometry.

From the "viewpoint of arithmetic", the geometrical dimension" of the separable complex Hilbert space should refer to infinity describing exhaustively any infinite set in a unique and relevant way.

From the "viewpoint of geometry", the arithmetical dimension of Hilbert space is a degeneration from unit balls to points or the borderline case of a limit where the magnitude of the "unit" converges to zero.

Both viewpoints can be unified into a single information one by the concept of choice as follows:

\footnotetext{
${ }^{7}$ Other fields of interpretation, properly philosophical, are also possible, e.g. Husserl's phenomenology was interpreted formally and mathematically as "quantum phenomenology" (Penchev 2021 July 26) or transcendentalism as "scientific transcendentalism" (Penchev 2021 October 20).

${ }^{8}$ The qubit Hilbert space replaces the "axes" of the separable complex Hilbert space by qubits as follows: the first and second axes constitute the first qubit; the second and the third axes constitute the second qubit; and so on: " $n$ " successive axes of the separable complex Hilbert space are transformed into "n-1" successive qubits. That transformation conserves unitarity in the qubit Hilbert space in relation to pairs of axes of the separable complex Hilbert space. The suggested construction of successive qubits by successive "axes" "decreases" the unitarity of the separable complex Hilbert space only with a bit (or two binary oppositions) in the following sense (Penchev 2021 July 8). Only the correspondence of the first and second axes to the first qubit cancels a bit of information (needing to be restored additionally). Then, the third axis corresponds unambiguously to the second qubit, the fourth axis, to the third qubit, etc.

${ }^{9}$ The geometrical aspect relevant to the quantity of quantum information as a unique measure for any infinite set can be considered according to the idea of "local space" in the Standard model. Correspondingly, the "global space" is meant by the antithetical aspectm, and both spaces are dual to each other and thus replaceable idempotently.
} 
the geometrical viewpoint is "before" the choice, and the arithmetic one, "after" $\mathrm{it}^{10}$. Information and quantum information mean the quantity of those choices, each of which is a single geometrical and arithmetical unit: correspondingly, "qubit" and "bit".

Both arithmetical and geometrical dimension share infinity but in different ways in a sense:

The arithmetical dimension implies rather the "potentially infinite" of an infinite constructive process "adding and adding units, one by one": the "principle of induction or transfinite induction".

The geometrical dimension implies rather the "actually infinite" as the result of the above process (the limit converging into a single point), but also the process as complete: as a whole. In fact, the concept of actual infinity means just this: the entire constructive process of potential infinity as complete to be "reduced" to its whole and eventually named as a single point in Hilbert space, i.e. the result, to which converges the potential constructive process in infinity.

The same property to link inherently potential and actual infinities can be deduced also from the "duality to the axiom of choice" and therefore, to the inherent duality of Hilbert space.

The next step to the foundation of mathematics originates immediately from the previous interpretation in terms of set theory for the latter is the standard basis of all mathematics unfortunately attacked by the Gödel (1931) "incompleteness theorems" in relation to the consistent completeness of arithmetic to it.

One can utilize a "mathematical metaphor" about the main theorem of algebra in order to elucidate the way for the main statement of this paper to be referred as to the foundation of mathematics. The main theorem of algebra finds the relevant set of numbers, which turns out to be the field of complex numbers, so that any polynomial equation with coefficients from this set possesses solutions, which are elements only of the same set.

The "scholia" is that one should search for that set, which is relevant to completeness for the granted one (e.g. that of real numbers in the case of the main theorem of algebra) can turn out to be insufficient.

Thus, arithmetic granted to be the necessary and self-obvious base for set theory and via it, to all mathematics is insufficient as the Gödel theorems demonstrate. One can search for that set, possibly including arithmetic, which is indeed relevant, independently of the prejudice.

The separable complex Hilbert space by its properties both to unify geometry and arithmetic and to name infinity in detail is a relevant applicant to be the basis of the cherished completeness and therefore, of the self-foundation of all mathematics.

The theorems about the absence of hidden variables in quantum mechanics (Neumann 1932; Kochen and Specker 1968 ${ }^{11}$ ) imply its completeness in the following sense: its eventual incompleteness might mean the existence of some unknown variables (so-called "hidden variables") relevant to what quantum mechanics studies but not available in its current formulation.

Einstein, Podolsky, and Rosen (1935) deduced the necessary existence of new physical phenomena (which are now called "phenomena of entanglement") from its mathematical formalism. They believed that their argument forced quantum mechanics to decide: either those phenomena cannot be confirmed experimentally and thus quantum mechanics was to be complemented in a way to exclude them as an absurd corollary (as Einstein suggested properly); or they can be corroborated and then

${ }^{10}$ Which is which is rather conventional for the state "before the choice" and that "after the choice" are interchangeable.

${ }^{11}$ The year of publishing the same paper is sometimes cited as "1967" according to the year when it has been accepted for publishing in the journal. 
quantum mechanics again was to be complemented though in a way to describe them exhaustively.

History realizes the latter alternative: (1) entanglement is confirmed very well experimentally, and even constitutes a corresponding science (relative to quantum mechanics), the theory of quantum information (or briefly, only "quantum information" or even, "quantum mechanics and information"). Nonetheless, (2) quantum mechanics is not incomplete (as the three authors believed to follow in the framework of the dilemma constructed by them) though (3) their inference is absolutely correct logically. The statements in this paragraph seem to be inconsistent to each other therefore qualified as a "paradox" sometimes (known also as the "EPR paradox") or needing an elucidation of the way how they can be consistent to each other.

The present paper (as well as many others) advocates that all phenomena of entanglement can be described by means of their "projections" on the inherently (or definitively) complete quantum mechanics as quantum correlations (able to exceed any correlations admissible in classical physics: a sufficient condition of which is the "violation of Bell's inequalities"). So, the last paragraph above is resolved without being a logical antinomy as follows: (1) \& (3) does not contradict (2) since quantum correlations are out of the scope only of classical physics rather than out of quantum mechanics ${ }^{12}$.

Having a careful look at the way for the completeness of quantum mechanics to be deduced, one notices that it depends only on the mathematical structure of the separable complex Hilbert space once it is established to be relevant as fundamental and underlying the entire building of quantum mechanics. Thus, the completeness of quantum mechanics can be considered as a property of the separable complex mechanics and independently of its physical interpretation. Then, one can say that separable complex (respectively, qubit) Hilbert space is inherently a complete mathematical structure, and Hilbert arithmetic is only a way to emphasise its special status in the foundation of mathematics.

If all the infinite sets are reliably differentiated from each other by their "wave function", this does not allow for identifying different infinities due to the uncertainty of the term "infinity" as a shared quality, but not admitting the clear distinguishability of arithmetic to natural numbers. The idea for the Gödel incompleteness to be overcome is the following:

If one considers the Gödel number of the so-called first incompleteness theorem ("Satz VI", Gödel 1931: 187), it turns out to be infinite necessarily as to set theory because its statement includes all positive integers explicitly ${ }^{13}$. Consequently, its negation will share the "same" infinite number. Thus, the theorem presupposes the incompleteness, which it will infer by its proof further. One can admit

\footnotetext{
${ }^{12}$ Anyway, the same "state of affairs" can be interpreted differently. One can ascribe classical correlations to all being "projectable temporarily" and consequently, the area of quantum correlations is to be out of time, i.e. really "spooky" (following Einstein's pictorial phrase, "spooky action at a distance"). Then, that area would be observable on the temporal "screen" of classical physics (and even, on that of the "classical" quantum mechanics within the framework of the Standard model) as the inexplicable "dark matter" and "dark energy" (Penchev 2021 October 20).

${ }^{13}$ In fact, still the concept of all natural numbers is contradictory in a theorem (such as "Satz VI") including simultaneously arithmetic (thus, the axiom of induction) and set theory (thus, the axiom of infinity). Indeed, the axiom induction implies for natural numbers to be finite, but the axiom of infinity states that the set of all finite numbers is infinite. Gödel's theorem at issue (or his paper as a whole) means all natural numbers simultaneously in relation to both set theory and arithmetic therefore predetermining the (inferred by him) dichotomy of the relation of arithmetic to set theory: either incomplete or contradictory. Its basis is the dichotomy of "all natural numbers" if they are considered simultaneously to both arithmetic and set theory: being finite in the former. they imply for it to be either incomplete (as a true subset in relation to the set itself) or contradictory (as a true subset identified with the set itself rather than only mapped by a bijection, since the latter is admissible or even definitive for infinitive sets). So, the Gödel number of the Gödel proposition including all natural numbers can be simultaneously finite in arithmetic, but infinite in set theory.
} 
that a special kind of that statement "liar" stating the own unprovability and fundamental for the Gödel theorems forces the theorem proved by its assistance to share an inherent uncertainty.

That kind of incompleteness or uncertainty can be avoided by ascribing different wave functions for any meta-statements possessing the "same infinite numbers" just as their negations ${ }^{14}$.

One can also conclude that the completeness of the separable complex Hilbert space, being inherently intrinsic, addresses some kind of neo-Pythagorean ontology (Penchev 2021 August 24). Quantum mechanics, though a doctrine about nature, but underlain by that Hilbert space is also an evidence of the same kind of fundamental mathematical ontology (being quite different from only the "ontology of mathematics" as the latter has been understood in the framework of Modernity).

Information theory is the next area of interpretation. Information as a quantity can be seen at least in two quite different ways: (1) as some kind of relations between two real functions (e.g. as the functional of the one in the basis of the other) interpretable as the entropies of any two states of a single system or as those of two systems; and (2) as some binary series interpretable both as a Turing machine and as an algorithm able to transform the one of the above states or systems into the other.

A few comments seem to be necessary:

1. The mathematical form of entropy is identical to that of expectation (representing a particular case of mathematical expectation; the expectation of the probability distribution itself). Consequently, entropy can be interpreted in two ways sharing one and the same formula and even mathematical structure: (1) as the entropy (in a proper sense) of a real state of some statistical ensemble; (2) as the mathematical expectation of the same real state.

The probabilities will be "objective" in the former case and "subjective" otherwise. However, they as being "subjective" as being "objective" are not more than different interpretations of the same formula therefore hinting at some hidden common "state of affairs" underlying both cases.

The separable complex Hilbert space is what generalizes that twofold interpretation (including as to any element of it).

The concept of information, thus, allows for a common quantity representing a relation of entropy in a proper sense and expectation. This means a quantity shared by present and future states.

2. One can be tempted to add the past to the range of information. This can happen if the past is axiomatized mathematically as well-ordering, and therefore as some number series reducible to binary.

3. Summarizing the above two considerations, one can conclude that the main statement interpreted in terms of information theory means generalizing the concept of information also to the past extending the invariance of description of entropy and expectation as to well-ordered number series. One can simply say:

Information is invariant to time, to past, present, and future, therefore being one of the most fundamental quantities both physical and mathematical ${ }^{15}$.

4. The main statement can be also interpreted in the framework of the same generalization, which extends the range of the equivalence of both Kolmogorov and entropic informations from finite to infinite series and statistical ensembles

4.1. Defined as a Turing machine or as an arbitrary algorithm, information can be immediately

\footnotetext{
${ }^{14}$ If one means Hilbert arithmetic, they should be the "same" (in the sense of dual counterparts), but belonging to the two dual qubit Hilbert spaces.

${ }^{15}$ Thus, information as quantum information can serve as the basis of the "conservation of energy conservation" in the "classical" quantum mechanics, but furthermore it is consistent with its violation in the more general framework of quantum information conservation (Penchev 2020 October 5).
} 
referred only to finite series.

4.2. Defined as entropic information, it is implicitly referred also only to finite statistical ensembles nevertheless that the approximating real functions address the continuum, on which they are dedined. That continual infinity is not inherent to the finite statistical ensemble, to which entropic information refers.

4.3. Unlike these "classical cases", both quantum information and transfinite ordinals are directly related to infinity.

This is obvious even in definition, as to the transfinite ordinals.

Quantum information being a quantity measurable by qubits addresses also inherently infinity as soon as a qubit can be interpreted as a choice among the elements of an infinite set.

Quantum mechanics being the "fatherland" of quantum information allows of interpreting its main equation, the Schrödinger equation, as a physical exemplification of the main statement:

One can suggest that the Schrödinger equation is well-known in all modifications. So, it will be sufficient and more suitable to describe it only qualitatively for the present objective:

It equates the first time derivative (therefore the change in time) of the wave function (therefore a value of quantum information) of any quantum system to the algebraic sum of (1, with sign minus sign) the second space derivative (laplacian and thus the tensor product of two changes in space) of the same wave function (and the same value of quantum information) corresponding to kinetic energy in classical mechanics and of (2, with a plus sign) the product of a potential function and the same wave function, corresponding to potential energy in classical mechanics.

Both sides of the equation have the physical dimension of energy. The coefficients involved in the equation are the Planck constant, the constant of light speed in a vacuum, the mass of the system (more exactly, the reduced mass of the system), and the imaginary unit, $i$, which is very important for the information interpretation of the equation.

A few preliminary comments are necessary:

1. The Schrödinger equation is a generalization of the fundamental law of energy conservation in classical mechanics: indeed, the former implies the latter if the wave function is a constant as a particular case. In turn, the generalization involves just complex functions (such as wave functions). One may suggest then that quantum information corresponds to a relevant generalization of energy already definable as to complex values therefore involving a second, i.e. just imaginary dimension of energy. That dimension can be thought of by the metaphor of "time energy" unlike kinetic and potential energy both being "space energies".

A suitable term is "space-time energy" as relevant to quantum information. Another option for them is to be designated correspondingly as temporal and spatial energy. Then, one of the possible meanings of the Schrödinger equation would be their equating. Furthermore, the spatial energy would correspond to quantum information defined as a statistical ensemble, and the temporal energy, defined as a transfinite ordinal therefore involving a Pythagorean identification of the physical and mathematical immediately and directly.

2. The potential and kinetic energy are clearly distinguished from each other in classical mechanics: kinetic energy corresponds to the real motion depending only on its velocity, and potential energy describes the force in each spatial point acting on a tentative reference unit (e.g. a body or a material point) depending only on its position. The law of energy conservation means the way for the acting force to be transformed into the motion of some real item studible by physics. The Schrödinger equation generalizes that classical understanding as follows: 
Wave function corresponds to space in a different way in the cases of kinetic and potential energy: The tensor product of two identical and complementary (or "conjugate") wave functions is mapped into space if the energy is kinetic, and a single wave function is mapped into space in the case of potential energy. Indeed the latter can be interpreted as the action on an energy- momentum unit thus depending only on the spatial coordinates.

However, this representation closer to the ideas of classical mechanics is "paraphrased" at present (and particularly in the Standard model) as a few kinds of fundamental symmetries: any symmetry is a relation between a set of symmetric elements as different and the same set as a single element being a class of equivalence to the symmetry in question.

Thus, the concept of symmetry expresses differently, but equivalently the underlying idea of quantum information: the choice of an element among an infinite set of equivalent, but different alternatives.

Nevertheless, the form of the Schrödinger equation is universal as it refers to all possible symmetries rather than to the ones really observed in experiments until now or to the ones included in the Standard model, namely: U(1), SU(2), and SU(3).

3. The invariance of discrete leaps and smooth motions allows for the Schrödinger equation to be represented by the corresponding units: bits and qubits. Furthermore, one can introduce a new distinction between real (or kinetic) and potential qubits: the former for kinetic energy, the latter for potential energy. Then, the Schrödinger equation can be formulated so:

4. The energy of a "(trans)finite bit" is equal to the subtraction of the energy of a potential and a kinetic qubit. The necessary equivalence of potential and kinetic qubits is implied by the equivalence of the probabilistic (Born 1927) and many-worlds interpretation (Everett III, 1957) and in turn implies the equivalence of a bit of a transfinite position ("transfinite bit") and a bit of a finite position ("finite bit").

5. The Kochen - Specker (1968) theorem helps us to avoid a mess:

The equation of the energies as above does not mean that a qubit can be equivalent to a bit, which would contradict to the direct corollary (Kochen, Specker 1968: 70), which is immediately after the main theorem of their paper (ibidem, a few lines above). In fact the concept of energy conservation (only generalized in the Schrödinger equation), as Emmy Noether's (1918) fundamental theorem shows, implies the equality of all moments of time.

Consequently, the equation of energies implies that the past moment corresponding to a bit, the present moment, to a kinetic qubit, and the future moment, to a potential qubit are already presupposed as equivalent, but this does not refer to the bits and qubits themselves: They are fundamentally different, and only the quantity of their corresponding energy can be equated.

Summarizing all considerations above, one can suggest the following meaning of the Schrödinger equation in terms of quantum information, It generalizes energy conservation to past, present, and future moments of time rather than only to present and future moments as this does the analogical law in classical mechanics, and therefore it describes the "appearance or occurrence of time", speaking loosely or metaphorically.

On the other hand, it can be considered as the universal law of how "time flows", or in other words, about the course of time (resolving by its course the main problem of quantum mechanics: the unification and uniform description of leap-like, properly quantum changes and smooth changes of the apparatus studied by classical mechanics). Furthermore, resolving its problem, the Schrödinger equation suggests the proportionality (or even equality if the units are relevantly chosen) of the 
quantities of both quantum and classical information and energy therefore being a (quantum) information analogue of Einstein's famous equality of mass and energy (" $E=m c^{2}$ ").

\section{CONCLUSIONS \& DIRECTIONS}

This paper shows how a few concepts in separated scientific areas can be synthesized by a single statement. Its proof is technically almost trivial, and thus it is interesting from a philosophical rather than properly mathematical viewpoint.

The main statement establishes the link between a transfinite number assignable to some infinite set and an element of the separable complex (or "qubit") Hilbert space interpretable both as a "wave function" in terms of quantum mechanics and as a given value of quantum information.

It allows further at least four quite different scientific fields (set theory, the foundation of mathematics, information theory, and quantum mechanics) to be synthesized in order to be considered as four interpretations of a single underlying mathematical structure, that of the separable complex Hilbert space.

That synthesis in turn addresses far-reaching speculations about ontology, epistemology, and other branches of philosophy due to the following reason. Quantum mechanics is a fundamental and scientific doctrine about nature; set theory and the foundation of mathematics underlie mathematics; and information theory is implemented as an essential part of contemporary techniques.

If there is a "bridge" for the direct, one-to-one interpretation between them, this implies some kind of neo-Pythagorean ontology making related mathematics, physics, and technics immediately, by an explicit mathematical structure.

Number and Being (by the meditation of time), the natural and artificial turn out to be not more than different hypostases of a single common essence.

The following directions can be outlined:

1. The "translations" of results between the aforesaid four areas: indeed, each of them contains unique results, which can be multiplied "by four" (Penchev 2021 August 24; Penchev 2021 July 26; Penchev 2021 October 20; Penchev 2020 August 25; Penchev 2021 April 12).

2. The research of other scientific areas is able to be "annexed" as far as they share the same or similar mathematical structure or for close links to the enumerated scientific areas (Penchev April 6; Penchev 2021 March 9; Penchev 2021 February 25; Penchev 2020 December 14; Penchev 2020 October 20; Penchev 2020 August 5; Penchev 2020 July 8; Penchev 2019a).

3. The investigation of those properties of the underlying mathematical structure, which would allow for that wide-ranging synthesis and their generalization or specification addressing new implementations (Penchev 2021 July 8a; Penchev 2021 July 8b; Penchev 2021 July 1; Penchev 2020 October 5; Penchev 2020 August 25).

4. A proper philosophical analysis of those far-reaching consequences and aftereffects for philosophy and its branches (Penchev 2021 August 24; Penchev 2021 July 26; Penchev 2021 April 6; Penchev 2021 February 2; Penchev 2021 October 20; Penchev 2021 April 12; Penchev 2020 October 19; Penchev 2020 August 18; Penchev 2020 June 4; Penchev 2019b). 


\section{REFERENCES}

Aspect, A., Grangier, R., Roger, G. (1981) "Experimental Tests of Realistic Local Theories via Bell's Theorem," Physical Review Letters 47 (7): 460-463.

Aspect, A., Grangier, R., Roger, G. (1982) "Experimental Realization of Einstein-Podolsky-Rosen-Bohm Gedanken Experiment: A New Violation of Bell's Inequalities," Physical Review Letters 49 (2): 91-94.

Bell, J. (1964) “On the Einstein - Podolsky - Rosen paradox," Physics (New York) 1 (3): 195-200.

Born, M. (1927) "Physical aspects of quantum mechanics," Nature 119 (2992): 354-357.

Cantor, G. (1897) "Beiträge zur Begründung der transfiniten Mengenlehre (Zweiter Artikel)," Mathematische Annalen 49 (2): 207- 246.

Einstein, A. (1926) "Letter to Max Born, 16 December 1926," in: Albert Einstein Max Born Briefwechsel 1916 - 1955 (kommentiert von Max Born). München: Nymphenburger Verlagshandlung, 1969, pp. 129-130.

Einstein, A., Podolsky, B., Rosen, N. (1935) "Can Quantum-Mechanical Description of Physical Reality Be Considered Complete?" Physical Review 47 (10): 777-780.

Everett III, H. (1957) „Relative state” Formulation of Quantum Mechanics,” Reviews of Modern Physics 29 (3): 454-462.

Gödel, K. (1931) "Über formal unentscheidbare Sätze der Principia mathematica und verwandter Systeme I," Monatshefte der Mathematik und Physik 38 (1): 173-198.

Kochen, S. and Specker, E. (1968) "The problem of hidden variables in quantum mechanics," Journal of Mathematics and Mechanics 17 (1): 59- 87.

Kolmogorov, A. (1965) "Tri podkhoda k opredeleniyu "kolichestvo informatsii (Three approaches to the definition of the quantity of information)," Problemy peredachi informatsii 1 (1): 3-11 (in Russian; a publication in English: Kolmogorov, A. (1968) "Three Approaches to the Quantitative Definition of Information," International Journal of Computer Mathematics 2 (1-4): 157-168).

Kolmogorov, A. (1968) "Logical basis for information theory and probability theory," IEEE Transactions of Information Theory IT-14: 662-664.

von Neumann, J. (1923) "Zur Einführung der transfiniten Zahlen," Acta litterarum ac scientiarum Ragiae Universitatis Hungaricae Francisco-Josephinae, Sectio scientiarum mathematicarum 1 (4): 199-208.

von Neumann, J. (1932). Mathematische Grundlagen der Quantenmechanik, Berlin: Springer, pp. 167-173 (Chapter IV.2).

Noether, E. (1918) "Invariante Variationsprobleme," Nachrichten von der Gesellschaft der Wissenschaften zu Göttingen. Mathematisch-Physikalische Klasse 1918: 235-257.

Penchev, V. (2021 August 24) "Hilbert arithmetic as a Pythagorean arithmetic: arithmetic as transcendental," SSRN, https://papers.ssrn.com/sol3/papers.cfm?abstract_id=3909610 or https://dx.doi.org/10.2139/ssrn.3909610.

Penchev, V. (2021 July 26) "Quantum phenomenology as a "rigorous science": the triad of epoché and the symmetries of information," SSRN, https://dx.doi.org/10.2139/ssrn.3892039 or

https://papers.ssrn.com/sol3/papers.cfm?abstract id=3892039 .

Penchev, V. (2021 July 8a) "Quantity in Quantum Mechanics and the Quantity of Quantum Information," SSRN, https://papers.ssrn.com/sol3/papers.cfm?abstract id $=3878772$ or

https://dx.doi.org/10.2139/ssrn.3878772.

Penchev, V. (2021 July 8b) "Two bits less" after quantum-information conservation and their interpretation as "distinguishability / indistinguishability" and "classical / quantum," SSRN,

https://dx.doi.org/10.2139/ssrn.3873123 or https://papers.ssrn.com/sol3/papers.cfm?abstract id=3873123 .

Penchev, V. (2021 July 1) “The 'Non-causal Causality' of Quantum Information,” SSRN, https://papers.ssrn.com/sol3/papers.cfm?abstract id=3870329 or https://dx.doi.org/10.2139/ssrn.3870329 .

Penchev, V. (2021 April 12) "Both Classical \& Quantum Information; Both Bit \& Qubit: Transcendental Time. Both Physical \& Transcendental Time," SSRN, https://dx.doi.org/10.2139/ssrn.3823665 or 
https://papers.ssrn.com/sol3/papers.cfm?abstract id=3823665.

Penchev, V. (2021 April 6) "Modal History versus Counterfactual History: History as Intention," SSRN, https://papers.ssrn.com/sol3/papers.cfm?abstract id=3818767 or https://dx.doi.org/10.2139/ssrn.3818767.

Penchev, V. (2021 March 9) "The Generalization of the Periodic Table: The 'Periodic Table' of 'Dark Matter'," SSRN, https://papers.ssrn.com/sol3/papers.cfm?abstract id=3800823 or https://papers.ssrn.com/sol3/papers.cfm?abstract id=3800823.

Penchev, V (2021 February 25) "Natural Cybernetics of Time, or about the Half of any Whole," SSRN, https://papers.ssrn.com/sol3/papers.cfm?abstract id $=3750608$ or https://dx.doi.org/10.2139/ssrn.3750608 .

Penchev, V. (2020 December 14) "Natural Cybernetics and Mathematical History: The Principle of Least Choice in History," SSRN, https://papers.ssrn.com/sol3/papers.cfm?abstract id=3714119 or https://dx.doi.org/10.2139/ssrn.3714119.

Penchev, V (2020 October 20) "Two deductions: (1) from the totality to quantum information conservation; (2) from the latter to dark matter and dark energy," SSRN, https://dx.doi.org/10.2139/ssrn.3683658 or https://papers.ssrn.com/sol3/papers.cfm?abstract id $=3683658$.

Penchev, V. (2020 October 19) "The Case of Quantum Mechanics Mathematizing Reality: The 'Superposition' of Mathematically Modeled and Mathematical Reality: Is There Any Room for Gravity?' SSRN, https://papers.ssrn.com/sol3/papers.cfm?abstract id=3683342 or https://dx.doi.org/10.2139/ssrn.3683342 .

Penchev, V. (2020 October 5) "Quantum-Information Conservation. The Problem About 'Hidden Variables', or the 'Conservation of Energy Conservation' in Quantum Mechanics: A Historical Lesson for Future Discoveries," SSRN, https://papers.ssrn.com/sol3/papers.cfm?abstract id=3675319 or https://dx.doi.org/10.2139/ssrn.3675319.

Penchev, V. (2020 August 25) "The Relationship of Arithmetic As Two Twin Peano Arithmetic(s) and Set Theory: A New Glance From the Theory of Information," SSRN, https://dx.doi.org/10.2139/ssrn.3656179 or https://papers.ssrn.com/sol3/papers.cfm?abstract id=3656179.

Penchev, V. (2020 August 18) "The Indeterminist Objectivity of Quantum Mechanics Versus the Determinist Subjectivity of Classical Physics," SSRN, https://dx.doi.org/10.2139/ssrn.3653015 or https://papers.ssrn.com/sol3/papers.cfm?abstract id=3653015.

Penchev, V. (2020 August 5) "A Class of Examples Demonstrating that 'P $\neq$ NP' in the 'P vs NP' Problem," SSRN, https://papers.ssrn.com/sol3/papers.cfm?abstract id $=3647038$ or https://dx.doi.org/10.2139/ssrn.3647038.

Penchev, V. (2020 July 8) "Problem of the Direct Quantum-Information Transformation of Chemical Substance," SSRN, https://papers.ssrn.com/sol3/papers.cfm?abstract id=3627003 or https://papers.ssrn.com/sol3/papers.cfm?abstract id $=3627003$.

Penchev, V. (2020 June 4) "All Science As Rigorous Science: The Principle of Constructive Mathematizability of Any Theory," SSRN, https://papers.ssrn.com/sol3/papers.cfm?abstract id=3618926 or https://dx.doi.org/10.2139/ssrn.3618926/

Penchev, V. (2019a) "From the Principle of Least Action to the Conservation of Quantum Information in Chemistry: Can One Generalize the Periodic Table?" Chemistry: Bulgarian Journal of Science and Education 28 (4): 525-539.

Penchev, V. (2019b) "Why Anything Rather Than Nothing? The Answer of Quantum Mechanics," in: I. Mladenov, A. Feodorov (eds.), Non/Cognate Approaches: Relation \& Representation. Sofia: "Парадигма", pp. 151-172.

Penchev, V. (2005) “Квантовият компютьр: квантовите ординали и типовете алгоритмична неразрешимост," Philosophical Alternatives 14 (6): 59-71 (in Bulgarian).

Schrödinger, E (1935) "Die gegenwärtige situation in der Quantenmechanik," Die Naturwissenschaften 23 (48): 807-812; 23 (49): 823-828; 23 (50) 844-849. 
Shannon, C. (1948) "A Mathematical Theory of Communication," Bell System Technical Journal 27 (3): 379-423; 27 (4), 623-656.

Skolem, T. (1922) "Einige Bemerkungen zur axiomatischen Begründung der Mengenlehre," in: Matematika Kongressen i Helsingfors den 4-7 Juli 1922, Den femte skandinaviska matematika kongressen, Redogörelse, Akademiska Bokhandeln, Helsinki, 1923: pp. 217-237 (Skolem, T. (1929) "Über einige Grundlagenfrage der Mathematik," Skrifter Vitenskapsakademiet i Oslo I (4): 1-49.)

Whitehead, A. N., Russell, B, (any edition) Principia Mathematica, Vol. $2(* 153)$, Vol. $3(* 251)$.

Wiener, N. (1938) “The Homogeneous Chaos," American Journal of Mathematics 60 (4): 897-936.

Wiener, N. (1948) Cybernetics; or, Control and communication in the animal and the machine, Cambridge, Mass.: MIT Press. 